\title{
Intramuscular 17-hydroxyprogesterone caproate to prevent preterm birth among HIV-infected women in Zambia: study protocol of the IPOP randomized trial
}

Joan T. Price ${ }^{1,2^{*}}$ (D), Bellington Vwalika ${ }^{1,2}$, Bethany L. Freeman ${ }^{1}$, Stephen R. Cole ${ }^{3}$, Helen B. Mulenga ${ }^{4}$, Jennifer Winston ${ }^{1}$, Felistas M. Mbewe ${ }^{5}$, Elwyn Chomba ${ }^{6}$, Lynne M. Mofenson ${ }^{7}$, Dwight J. Rouse ${ }^{8}$, Robert L. Goldenberg ${ }^{9}$ and Jeffrey S. A. Stringer ${ }^{1}$

\begin{abstract}
Background: Each year, an estimated 15 million babies are born preterm, a global burden borne disproportionately by families in lower-income countries. Maternal HIV infection increases a woman's risk of delivering prematurely, and antiretroviral therapy (ART) may compound this risk. While prenatal progesterone prophylaxis prevents preterm birth among some high-risk women, it is unknown whether HIV-infected women could benefit from this therapy. We are studying the efficacy of progesterone supplementation to reduce the risk of preterm birth among pregnant women with HIV in Lusaka, Zambia.

Methods: The Improving Pregnancy Outcomes with Progesterone (IPOP) study is a Phase III double-masked, placebo-controlled, randomized trial of intramuscular 17-alpha hydroxprogesterone caproate (17P) to prevent preterm birth in HIV-infected women. A total of 800 women will be recruited prior to 24 weeks of gestation and randomly allocated to 17P or placebo administered by weekly intramuscular injection. The primary outcome will be a composite of live birth prior to 37 completed gestational weeks or stillbirth at any gestational age. Secondary outcomes will include very preterm birth $(<34$ weeks), extreme preterm birth $(<28$ weeks), small for gestational age $(<10$ th centile), low birth weight $(<2500 \mathrm{~g})$, and neonatal outcomes. In secondary analysis, we will assess whether specific HIV-related covariates, including the timing of maternal ART initiation relative to conception, is associated with progesterone's prophylactic efficacy, if any.

Discussion: We hypothesize that weekly prenatal 17P will reduce the risk of HIV-related preterm birth. An inexpensive intervention to prevent preterm birth among pregnant women with HIV could have substantial global public health impact.
\end{abstract}

Trial registration: NCT03297216; September 29, 2017.

Keywords: Preterm birth, Progesterone, 17-alpha hydroxyprogesterone caproate, HIV, Antiretroviral therapy, SubSaharan Africa

\footnotetext{
* Correspondence: Joan_Price@med.unc.edu

'Division of Global Women's Health, Department of Obstetrics and

Gynecology, University of North Carolina at Chapel Hill, 3009 Old Clinic

Building, Campus Box 7577, Chapel Hill, NC 27599-7577, USA

${ }^{2}$ Department of Obstetrics and Gynaecology, University of Zambia School of

Medicine, Lusaka, Zambia

Full list of author information is available at the end of the article
}

(c) The Author(s). 2019 Open Access This article is distributed under the terms of the Creative Commons Attribution 4.0 International License (http://creativecommons.org/licenses/by/4.0/), which permits unrestricted use, distribution, and reproduction in any medium, provided you give appropriate credit to the original author(s) and the source, provide a link to the Creative Commons license, and indicate if changes were made. The Creative Commons Public Domain Dedication waiver (http://creativecommons.org/publicdomain/zero/1.0/) applies to the data made available in this article, unless otherwise stated. 


\section{Background}

Each year worldwide nearly 15 million babies are born prior to 37 weeks of gestation, of whom 1 million die as a consequence of prematurity [1]. The burden of preterm birth (PTB) and its associated mortality and long-term disability is disproportionately borne by the world's poorest families. More than $60 \%$ of global preterm deliveries occur in South Asia and sub-Saharan Africa, where resources to care for premature newborns are scarce and case fatality is high [2]. The geographic disparity in rates of prematurity may in part reflect the distribution of maternal HIV, which increases the risk of PTB [3]. Of 1.5 million women living with HIV who become pregnant each year, the overwhelming majority reside in either sub-Saharan Africa (87\%) or South Asia (5\%) [4]. While expanding coverage of antiretroviral therapy (ART) among pregnant women living with HIV has drastically reduced the incidence of mother-to-child transmission, maternal ART exposure does not appear to ameliorate the increased risk of PTB in HIV-infected pregnant women [5-10]. Additionally, neonatal mortality remains elevated in HIV-infected pregnant women on ART compared to HIV-uninfected women; in a study comparing women on efavirenz or dolutegravir-based ART, neonatal mortality was $2.3 \%$ among HIV-infected compared to $1.4 \%$ among HIV-uninfected women [9]. A considerable proportion of this neonatal mortality appears to be secondary to PTB [6].

Prenatal progesterone reduces the risk of preterm delivery in women who have had a prior spontaneous PTB and in those with sonographic evidence of cervical shortening in the mid-trimester. It is standard of care in the United States for these indications [11]. A 2013 Cochrane meta-analysis of progesterone to prevent PTB among women reporting a prior PTB aggregated data from 10 randomized trials studying prenatal prophylaxis by the intramuscular (IM, $n=4$ studies), vaginal ( $n=5$ studies), or oral ( $n=1$ study) route and estimated the risk ratio of birth prior to 37 weeks among women receiving active drug to be 0.55 (95\% CI: 0.42, 0.74) [12].

We seek to determine whether 17-hydroxyprogesterone caproate (17P) will reduce the risk of PTB among HIV-infected pregnant women receiving ART. Here we present the study protocol for a randomized trial designed to answer this question.

\section{Methods}

\section{Study design}

The Improving Pregnancy Outcomes with Progesterone (IPOP) trial is a double-masked, placebo-controlled, randomized trial of 17P to prevent PTB among HIV-infected women in Zambia. It is registered with clinicaltrials.gov under identifier: NCT03297216. Participants are randomly assigned to weekly intramuscular administration of either
17P or placebo manufactured to be indistinguishable started between 16 and 24 weeks gestational age. The study's primary outcome is a composite measure of delivery prior to 37 weeks or stillbirth at any gestational age. IPOP is being conducted in the antenatal clinics of the Kamwala District Health Centre (KDHC) and Women and Newborn Hospital of the University Teaching Hospital (WNH-UTH) in Lusaka. We also recruit participants from other public sector facilities in Lusaka. The IPOP trial has been designed following the Consolidated Standards of Reporting Trials (CONSORT 2010) Statement and the Standards for Protocol Items: Recommendations for Interventional Trials (SPIRIT 2013) (Fig. 1) [13, 14].

\section{Study participants}

Women meeting the following inclusion criteria are eligible to participate in the IPOP study: (1) 18 years of age

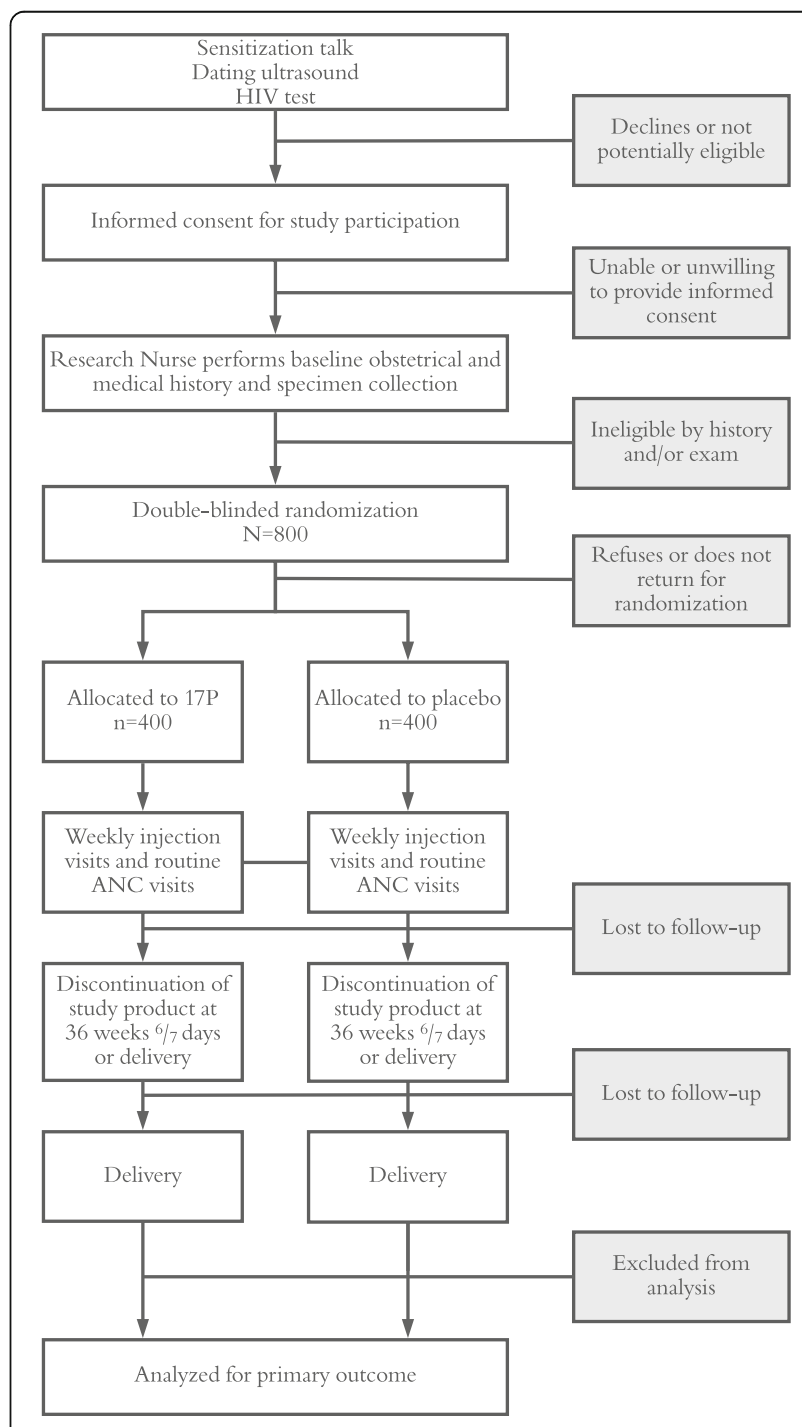

Fig. 1 IPOP study participant flow diagram 
or older; (2) viable intrauterine singleton pregnancy confirmed by ultrasound; (3) less than $24 \% / 7$ weeks of gestation; (4) antibody-confirmed HIV-1 infection; (5) currently receiving ART or intending to commence ART in pregnancy; (6) ability and willingness to provide written informed consent; (7) intent to remain in current geographical area of residence for the duration of study; and (8) willing to adhere to weekly study visit schedule. We exclude from participation any woman with the additional exclusion criteria: (1) confirmed prior spontaneous preterm birth; (2) multiple gestation; (3) known uterine anomaly; (4) planned or in situ cervical cerclage; (5) major fetal anomaly detected on screening ultrasound; (6) indication for planned delivery prior to 37 weeks (e.g. prior classical cesarean); (7) evidence of threatened abortion, preterm labor, or ruptured membranes at time of enrollment; (8) known allergy or medical comorbidity listed as a contraindication to $17 \mathrm{P}$ in the prescribing information; (9) prior participation in the trial; and (10) any other condition (social or medical) which, in the opinion of the study staff, would make trial participation unsafe or complicate data interpretation.

Current Zambia treatment guidelines recommend ART initiation for all individuals living with HIV, including pregnant and breastfeeding women [15]. The current ART regimen for first-line therapy for pregnant women living with HIV in Zambia is combination tenofovir disoproxil fumarate, lamivudine and efavirenz, with second-line therapy being combination zidovudine, lamivudine and a protease inhibitor, lopinavir-ritonavir or atazanavir-ritonavir; most pregnant women are receiving first-line ART.

\section{Intervention}

Trial participants are randomly allocated to one of two treatment groups in a 1:1 ratio. The first randomization group (17P) receives weekly $1 \mathrm{~mL}$ injections of $250 \mathrm{mg}$ 17-hydroxyprogesterone caproate while the other group (control) receives placebo manufactured to be indistinguishable, also by injection.

Study product (both active drug and placebo) is produced by AMAG Pharmaceuticals of Waltham, MA, USA, under the brand name of Makena ${ }^{\circ}$. Packaged and labeled product is shipped to each study site and dispensed to the administering nurse by an on-site pharmacist or pharmacy technician. Pharmacy staff have no direct contact with participants or potential participants, and are the only staff in Zambia not masked to treatment allocation. Participants begin weekly administration of study product from the day of randomization (between $16 \% / 7$ and $23 \% / 7$ gestational weeks, inclusive) until $36 \% / 7$ gestational weeks, stillbirth or delivery, whichever is sooner.

If an adverse drug reaction is identified in any participant receiving injections of study product, staff will consult with investigators to determine whether the reaction requires temporary or permanent discontinuation of study product based on the severity of the event and whether or not the event resolves. Study product will be immediately and permanently discontinued for any participant who has an anaphylactic reaction. The investigator will decide whether to un-blind the treatment group to guide treatment and future care for the participant. In general, adverse drug reactions will typically not require un-blinding of study group and will not necessarily indicate that a participant is receiving the active product since components in the placebo base may also result in adverse reactions.

\section{Objectives}

The primary objective of IPOP is to evaluate - among HIV-infected pregnant women receiving ART - whether $17 \mathrm{P}$ will reduce the risk of the composite outcome (live birth prior to 37 weeks of gestation or stillbirth at any gestational age) relative to placebo. Secondary objectives include: (1) to assess, through a subgroup analysis, whether 17P will reduce the risk of the primary outcome among women either (a) newly initiating ART or (b) continuing ART that was started prior to conception; (2) to assess the effect of timing of ART initiation on risk of the composite primary outcome and its components by comparing women newly starting ART to those who initiate ART prior to conception (a non-randomized comparison); and (3) to assess the extent to which exclusion of non-inflammatory PTB phenotypes from our outcome definition modifies the efficacy of $17 \mathrm{P}$ to prevent the composite primary outcome and/or any association between timing of ART initiation and the composite primary outcome. Finally, our tertiary objective is to investigate the underlying biology of PTB and other adverse pregnancy outcomes with particular attention to systemic (peripheral blood) inflammation and immune activation, local (vaginal) inflammation, and alterations of the vaginal microbiota.

\section{Study procedures}

Our recruitment activities include community sensitization in the catchment area of the recruitment clinics to educate community members about the trial and encourage early presentation for antenatal care. In addition, study staff conduct health talks at the recruitment clinics focusing on the importance of early and complete antenatal care, possible prevention of preterm delivery, and study inclusion and exclusion criteria.

Study staff identify potential participants attending antenatal care prior to 24 estimated gestational weeks (Table 1). An ultrasound is conducted to confirm potential study eligibility and, among women between 16 and 24 weeks of gestation, cervical length by transvaginal 
Table 1 IPOP study schedule of evaluations

\begin{tabular}{|c|c|c|c|c|c|c|c|c|}
\hline Visit Number & 0.0 & 1.0 & 2.0 & 3.0 & 4.0 & 5.0 & 6.0 & 7.0 \\
\hline Gestational Age (weeks) & $<24$ & $16-23$ & 24 & 28 & 32 & 36 & Delivery $^{c}$ & 42 days \\
\hline \multicolumn{9}{|l|}{ Administrative/regulatory procedures } \\
\hline Informed consent & $\bullet$ & & & & & & & \\
\hline Confirmation of eligibility & & $\bullet$ & & & & & & \\
\hline Collection/review of locator info & $\bullet$ & $\bullet$ & $\bullet$ & $\bullet$ & $\bullet$ & $\bullet$ & $\bullet$ & $\bullet$ \\
\hline Randomization & & $\bullet$ & & & & & & \\
\hline \multicolumn{9}{|l|}{ Clinical/behavioral procedures } \\
\hline Ultrasound ${ }^{\mathrm{a}}$ & $\bullet$ & $\bullet$ & & & $\bullet$ & & & \\
\hline Obstetrical history & $\bullet$ & & & & & & & \\
\hline Medical history and clinical exam ${ }^{a}$ & $\bullet$ & & $\bullet$ & & $\bullet$ & $\bullet$ & $\bullet$ & $\bullet$ \\
\hline Concomitant medication assessment & $\bullet$ & & $\bullet$ & & $\bullet$ & $\bullet$ & $\bullet$ & $\bullet$ \\
\hline Demographic history & $\bullet$ & & & & & & & \\
\hline Behavioral and nutritional assessment & $\bullet$ & & & & & & & $\bullet$ \\
\hline Maternal depression screen & $\bullet$ & & & & & & & $\bullet$ \\
\hline Infant clinical assessment & & & & & & & $\bullet$ & $\bullet$ \\
\hline \multicolumn{9}{|l|}{ Study product procedures } \\
\hline 17P Adherence counseling ${ }^{b}$ & & $\bullet$ & $\bullet$ & $\bullet$ & $\bullet$ & $\bullet$ & & \\
\hline Study drug injections ${ }^{\mathrm{b}}$ & & $\bullet$ & $\bullet$ & $\bullet$ & $\bullet$ & $\bullet$ & & \\
\hline Side effects assessment ${ }^{b}$ & & & $\bullet$ & $\bullet$ & $\bullet$ & $\bullet$ & & \\
\hline \multicolumn{9}{|l|}{ Laboratory procedures } \\
\hline Maternal rapid HIV ${ }^{a}$ & $\bullet$ & & & & & & & \\
\hline Maternal pregnancy test ${ }^{a}$ & $\bullet$ & & & & & & & \\
\hline Maternal rapid syphilis ${ }^{a}$ & $\bullet$ & & & & & & & \\
\hline Maternal candida, gram stain ${ }^{a}$ & $\bullet$ & & & & & & & \\
\hline Maternal hemoglobin (HemoCue) ${ }^{a}$ & $\bullet$ & & & & $\bullet$ & & & \\
\hline Maternal urinalysis ${ }^{a}$ & $\bullet$ & & & & $\bullet$ & & & \\
\hline Maternal viral load and T cell assays & $\bullet$ & & $\bullet$ & & & & $\bullet$ & \\
\hline Placenta, membranes, cord blood storage & & & & & & & $\bullet$ & \\
\hline Infant HIV DNA PCR & & & & & & & & $\bullet$ \\
\hline Vaginal-rectal swab storage & $\bullet$ & & $\bullet$ & $\bullet$ & & & $\bullet$ & \\
\hline Blood storage & $\bullet$ & & $\bullet$ & $\bullet$ & & & $\bullet$ & \\
\hline Urine storage & $\bullet$ & & $\bullet$ & $\bullet$ & & & $\bullet$ & \\
\hline
\end{tabular}

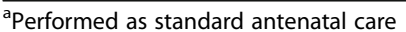

${ }^{b}$ Occurs weekly through week 36 , or until delivery, whichever occurs first

${ }^{c}$ All procedures may not be completed for women who deliver off-hours or in a location without staff coverage

ultrasound [16]. Those who meet preliminary eligibility by ultrasound and who agree to participation undergo a detailed informed consent process in English, Bemba, or Nyanja, depending on their language preference. After consent, study staff verify antenatal and HIV history data through a baseline questionnaire and review of participants' available medical records, perform a physical exam, and complete point of care testing for antenatal care and to confirm participants' HIV status.

Participants then undergo randomization into one of two treatment groups and start study drug as early as possible within the window of 16 and 24 weeks of gestation. Eligible women who choose to participate at the screening visit are given a subsequent appointment for randomization no earlier than the following business day to encourage careful consideration of the risks and benefits of study participation. On the day of randomization, following final confirmation of eligibility, participants are assigned with equal probability into one of two study groups. Our randomization scheme stratifies participants by timing of ART initiation (during current pregnancy / pre-pregnancy). A statistician from the UNC Center for 
AIDS Research Biostatistics Core not otherwise associated with the study created the scheme using random permuted blocks. At the time of randomization, a research nurse confirms eligibility in a web-based randomization program. Using the same web-based tool, the pharmacist then performs the randomization procedure and prepares the $1 \mathrm{~mL}$ injection of the assigned study treatment, which the research nurse administers to the participant. All randomization documentation is stored securely.

Once IPOP participants start study drug, they are seen weekly (Fig. 2). At each injection visit, study staff evaluate participants for side effects of IM progesterone, which are expected to be rare and generally mild, but include injection-site reactions, headache, breast tenderness, nausea, and cough. In addition, the study provides routine antenatal care to all participants following Zambian national guidelines at: 24, 32, and 36 gestational weeks.

At the 24- and 28-week study visits, blood, urine, and vaginal swabs are collected. At the time of delivery or shortly thereafter, blood, urine, and vaginorectal swabs are collected (Table 1). At delivery, study nurses collect samples of the placenta, umbilical cord, and cord blood. Also at delivery, study nurses obtain detailed information about the participant and her infant's clinical course by patient self-report, review of medical records, or by direct assessment. We assign a clinical phenotype [17] for all PTB and stillbirth outcomes as soon as possible following delivery. Finally, we conduct a routine 42-day postnatal visit, which includes clinical assessment of both mother and infant, and testing for infant HIV infection.

\section{Laboratory procedures}

Study staff obtain all samples from trial participants according to standard operating procedures. All samples are processed according to the assay manufacturers' specifications. Some specimens collected from patients in this protocol are analyzed immediately per standard antenatal care guidelines. Others are stored temporarily for later study-related analysis to investigate biological mechanisms contributing to preterm birth and other adverse birth outcomes.

\section{Study monitoring}

An external monitor will be contracted to oversee the progress of the clinical trial and to ensure that it is conducted, recorded, and reported in accordance with the protocol, standard operating procedures, International Conference on Harmonization / Good Clinical Practices, the Zambian National Health Research Act, and U.S. 45 CFR 46 requirements. The monitor will verify that the data reported is valid, accurate, and complete through review of case report forms, medical records, and source documentation. The monitor will make site visits each quarter through the end of the study and will provide verbal and written feedback to the study site for ongoing quality control and improvement.

We will constitute a Data Safety and Monitoring Board (DSMB) to periodically monitor trial performance and safety. The group will comprise senior investigators with statistical, methodological, and topical expertise who are not otherwise involved with the study. Their first charge will be to review the study protocol and create a monitoring schedule and stopping guidelines, based on expected event rates. Other specific duties will include: (1) periodic assessments of recruitment, accrual, retention, and data quality; (2) considering new external data as they come available, including scientific or therapeutic developments that may have an impact on participant safety or the ethics of the trial; (3) evaluation of scheduled interim analysis. Mortality outcomes will be reviewed by the DSMB during routine meetings. The DSMB will have authority to enjoin enrolment or stop the study altogether for reasons of patient safety. If an interim analysis were to show unequivocal benefit before follow-up were complete, we would support stopping the study and reprogramming remaining resources to make the intervention available to all participants.

\section{Primary outcome}

Our primary outcome is a composite of live birth occurring before 37 gestational weeks or stillbirth at any gestational age. Secondary outcomes are: delivery prior to 34 weeks of gestation and prior to 28 weeks of gestation; birth weight $<10$ th percentile for gestational age; birth weight $<3$ rd percentile for gestational age; mother-to-child HIV transmission by 6 weeks postpartum; cumulative incidences of the competing risks of stillbirth and PTB; neonatal and perinatal mortality rates; infant APGAR scores. Our tertiary outcomes are: birth weight $<2500 \mathrm{~g}$ and $<1500 \mathrm{~g}$; measures of maternal inflammation and/or immune activation; alterations of the vaginal microbiome; measures of infant morbidity (e.g., intensive care unit admission, supplemental oxygen requirement, need for assisted ventilation); and serious adverse events and events resulting in study product discontinuation.

\section{Sample size}

We estimated the baseline risk of the composite outcome (p1) as $24 \%$, based on estimates of preterm birth (21\%) plus stillbirth (3\%) among HIV-infected women in Zambia from our own local data $[18,19]$ and from modeled data [2]. We hypothesize that $17 \mathrm{P}$ will reduce the proportion of HIV-infected women experiencing the composite endpoint by $38 \%$ (from 24 to $15 \%$ ) [12]. A trial with 325 patients per group would have $80 \%$ power 


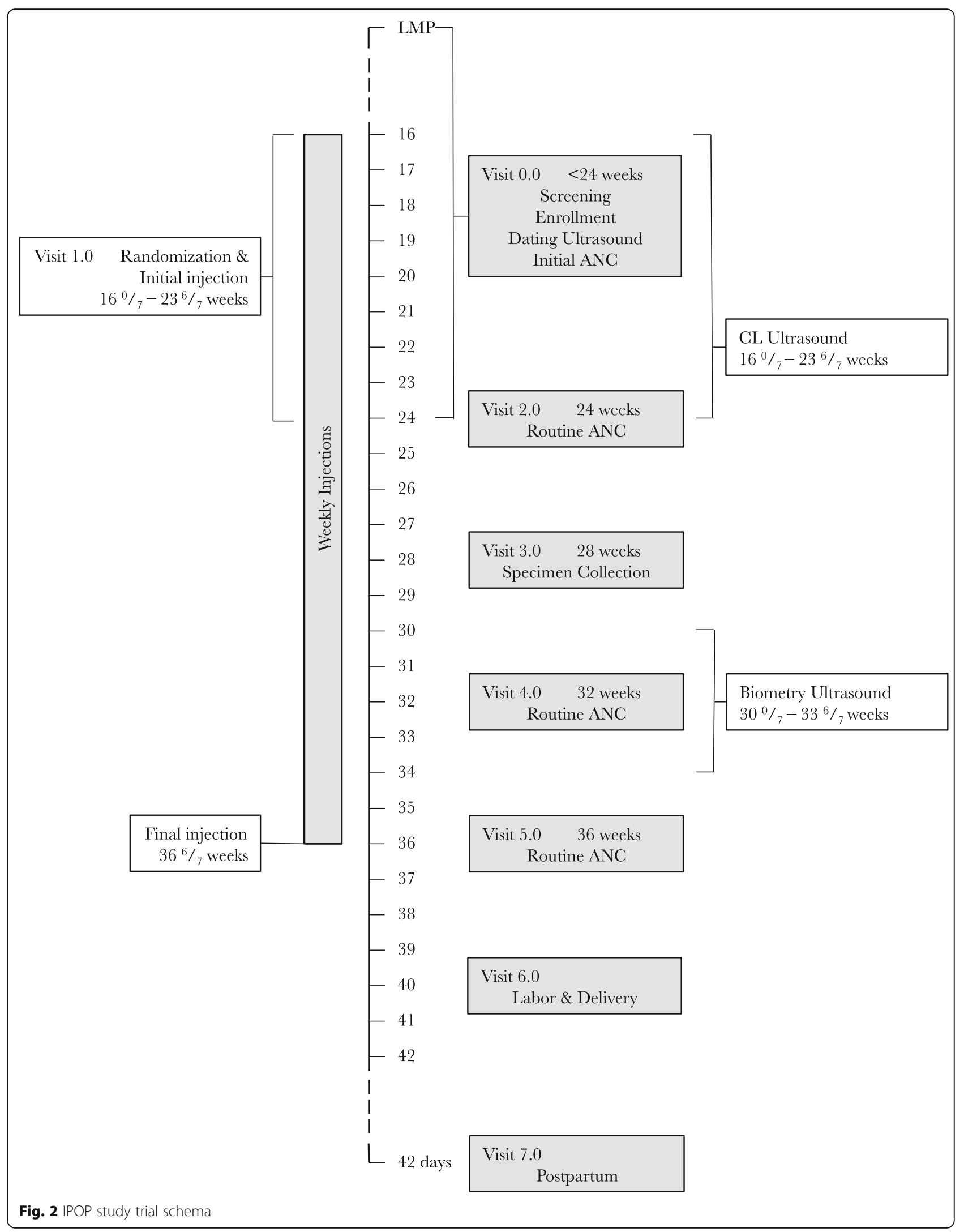


to detect this effect size (based on a 2-sided Fisher's exact test at the 0.05 significance level). To allow for up to $15 \%$ follow-up loss and to account for uncertainty in our estimates of baseline event probability (p1) and relative efficacy of the intervention, we set target enrollment at 400 women per randomization group in the trial.

\section{Statistical analysis plan}

We will assign an estimated delivery date (EDD) by ultrasound biometry measurements collected at time of enrollment, using the INTERGROWTH 21st standards $[20,21]$. We do not consider the participants' reported last menstrual period in our gestational age calculations, as this has been shown in our setting to introduce bias [22]. Randomized women who deliver (1) a live infant 21 or more days before their assigned EDD or (2) a stillborn infant at any gestational age will be categorized as having met the primary outcome.

The primary analysis will employ the intent-to-treat principle, wherein each mother-infant pair will be analyzed according to randomization assignment regardless of whether the mother complied with study procedures. The primary comparison between the two trial groups will be based on the estimated risk ratio of the primary outcome. The estimated risk of the primary outcome will be computed in each group using the Kaplan-Meier method to adjust for loss-to-follow-up, where mother-infant pairs who drop out of the trial prior to the primary outcome or live-birth of full-term infant will be right censored at their last study visit. A $Z$-statistic will be constructed by taking the difference in the estimated log risks divided by the corresponding estimated standard error (using the Greenwood estimator). As a sensitivity analysis, a Cox proportional hazards model with treatment assignment as the sole covariate will be fit to estimate the hazard of meeting the primary endpoint between randomization groups; the estimated hazard ratio is anticipated to be similar to the estimated risk ratio given that the primary outcome is relatively rare.

We plan to undertake a pre-specified subgroup analysis for this trial. This subgroup analysis will assess whether the effect of 17P differs within levels of the stratification factor. Effect modification will be assessed by fitting Cox proportional hazards models that include randomization group, the stratification factor, and a product term as covariates. A likelihood ratio test of the product term coefficient will be used to assess possible effect modification. If the effect modification tests are significant, hazard ratios will be estimated separately within levels of the factor (e.g., hazard ratios of 17P versus placebo for (i) mothers on pre-pregnancy ART and (ii) mothers initiating ART in pregnancy).

To assess the efficacy of 17P, a per-protocol analysis of the primary outcome will also be conducted that censors participants who fail to comply with their randomization assignment (defined here as missing two injections of study product in a row), and then reweights the data to account for possible informative censoring by noncompliance using inverse-probability (IP) weights [23]. We have previously used this and related methods to construct a consistent estimator of the per-protocol effect under the assumption that we have measured and correctly-adjusted for the common causes of noncompliance and the outcome [24-26]. The set of measured possible common causes includes demographic, medical, and obstetrical factors. We will construct IP weights using pooled logistic regression (pooling over time), with continuous variables fit using flexible restricted splines. We will explore the sensitivity to IP weight estimation [27].

\section{Ethical considerations}

Participation in this trial is voluntary. All participants undergo a comprehensive written informed consent process prior to study enrollment. Clinical study procedures are conducted according to local standards of routine care. All staff with direct participant contact receive training on protecting human research participants [28] before performing any study procedures and routinely thereafter. Key staff also undergo Good Clinical Practices training [29] no less frequently than every 3 years. The University of Zambia Biomedical Research Ethics Committee and the University of North Carolina at Chapel Hill Institutional Review Board each granted approval of the IPOP trial protocol before enrollment commenced.

Investigators make every reasonable effort to minimize risks to participants. We expect that participants will be exposed to minimal risk in this trial. Side effects and serious adverse events associated with 17P administration have been studied extensively and are rare [30]. Women with one or more contraindication to 17P that appear in the Makena ${ }^{\circ}$ package insert [31] are excluded from the trial. Physical risks of IPOP study participation also include the risks associated with venipuncture, which are infrequent and minimized with the use of proper technique. Collection of vaginal and rectal samples may also be associated with some discomfort or mild bleeding.

Participation in clinical research includes possible breach of confidentiality as well as discomfort with personal medical and socio-behavioral questions. Although investigators make every effort to protect participant privacy and confidentiality to reduce these risks (e.g., by conducting consent procedures in a private setting and do not include participant names on case report forms), it is possible that participant involvement in the study could become known to others, and that social harms may result (e.g., participants could become known as HIV-infected).

At each study visit, study staff assess participants for occurrence of social harms and adverse events. The 
severity of any study-related adverse events is graded based on the National Institute of Health's Division of AIDS Table for Grading the Severity of Adult and Pediatric Adverse Events [32]. Study staff report adverse events or social harms that are related to the study drug and/or study participation according to requirements by each individual regulatory authority.

To maintain participant confidentiality, all laboratory specimens, reports, study data, and administrative forms are identified by a coded number only. All databases are secured with password-protected access systems, and computer entries are identified by coded number only. Forms, lists, logbooks, appointment books, and any other listings or data forms that link participant identification numbers to other identifying information are stored in a separate, locked cabinet. All statistical analyses will use data identified only by the coded study number. Clinical information with individual identifiers will not be released without the written permission of the participant.

Participants who receive 17P in the IPOP trial may benefit from a reduced risk of delivering preterm if the intervention is indeed effective, but it is also possible that there will be no effect. Participants in both study groups may benefit from added health education and counseling, comprehensive antenatal care, and close follow-up. Findings from this study could shape health policy and clinical care, or inform future research in the prevention of HIV-related PTB. We intend to make all learning from this trial widely and quickly available by publishing in open access journals to facilitate informed decision-making by key stakeholders engaged in PTB prevention worldwide.

\section{Discussion}

The global epidemics of preterm birth and HIV converge in sub-Saharan Africa, where the skilled providers and resources needed to care for preterm neonates are scarce. Antiretroviral therapy for the prevention of mother-to-child HIV transmission has led to encouraging reductions in new cases of pediatric HIV, but despite preventing transmission this treatment does not appear to reduce the elevated risk of PTB and neonatal morbidity/mortality compared to HIV-uninfected pregnant women; in a study in Botswana $23 \%$ of women living with HIV on ART experience PTB compared to $15 \%$ of uninfected women [10]. In resource-limited settings where HIV complicates a considerable proportion of pregnancies, strategies to ameliorate PTB risk in women using ART could result in a substantial public health impact.

With this Phase III double-masked, placebo-controlled, randomized clinical trial, we aim to discover whether 17P will prevent PTB and stillbirth among HIV-infected women receiving ART in pregnancy. We will also, through planned sub-group analysis, gain insight into whether the effect of 17P varies between women initiating antiretroviral therapy prior pregnancy versus those newly starting treatment in pregnancy. Although pilot studies of vaginal progesterone to prevent PTB among HIV-infected women are ongoing $[33,34]$, the IPOP Study represents the first full-scale efficacy trial of antenatal progesterone in this population. If efficacious in this high-risk group, 17P has the potential to prevent hundreds of thousands of preterm births per year worldwide.

\section{Abbreviations \\ 17P: 17-alpha hydroxyprogesterone caproate; ANC: antenatal care; ART: Antiretroviral therapy; CONSORT: Consolidated Standards of Reporting Trials; DSMB: Data Safety and Monitoring Board; EDD: Estimated delivery date; HIV: Human immunodeficiency virus; IM: Intramuscular; IP: inverse probability; IPOP: Improving pregnancy outcomes with progesterone; KDHC: Kamwala District Health Centre; LMP: last menstrual period; PTB: Preterm birth; SPIRIT: Standard Protocol Items: Recommendations for Interventional Trials; UNC: University of North Carolina; WNH-UTH: Women and Newborn Hospital of the University Teaching Hospital}

\section{Acknowledgements}

Not applicable

\section{Funding}

The clinical trial presented in this article is funded through an award from the United States National Institutes of Health (R01 HD087119) and the Bill and Melinda Gates Foundation (OPP1172799). Trainee support has been provided through the National Institutes of Health (T32 HD075731 and K01 TW010857) for JTP. JSAS also receives support in part by the UNC Center for AIDS Research (P30A150410). The conclusions and opinions expressed in this article are those of the authors and do not necessarily reflect those of the National Institutes of Health or Bill and Melinda Gates Foundation. The funding bodies were not involved in the design, collection, analysis, and interpretation of data, nor in the writing of the manuscript.

\section{Availability of data and materials} Not applicable.

\section{Authors' contributions}

JTP, BLF and JSAS conceived of the study question and SRC, JTP, BLF, BV, FMM, EC, LMM, DJR, RLG and JSAS each contributed to study design. SRC and JW performed statistical analyses; JTP, BLF, HBM, JW and JSAS planned and coordinated randomization and pharmacy procedures. JTP, BLF, BV and JSAS each contributed to the writing of this manuscript. All authors critically reviewed and approved the final manuscript.

\section{Ethics approval and consent to participate}

This study was approved by the University of North Carolina Institutional Review Board, reference \#17-1173, and the University of Zambia Biomedical Research Ethics Committee, reference \#015-06-17, and the Zambia Medicines Regulatory Authority, reference CT-070. Written informed consent is obtained from all participants prior to study enrollment.

\section{Consent for publication}

Not applicable

\section{Competing interests}

The authors declare that they have no competing interests.

\section{Publisher's Note}

Springer Nature remains neutral with regard to jurisdictional claims in published maps and institutional affiliations. 


\section{Author details}

'Division of Global Women's Health, Department of Obstetrics and Gynecology, University of North Carolina at Chapel Hill, 3009 Old Clinic Building, Campus Box 7577, Chapel Hill, NC 27599-7577, USA. ${ }^{2}$ Department of Obstetrics and Gynaecology, University of Zambia School of Medicine, Lusaka, Zambia. ${ }^{3}$ Department of Epidemiology, University of North Carolina at Chapel Hill, Chapel Hill, NC, USA. ${ }^{4}$ Pharmaceutical Society of Zambia, Lusaka, Zambia. ${ }^{5}$ UNC Global Projects - Zambia, Lusaka, Zambia.

${ }^{6}$ Department of Paediatrics, University Teaching Hospital, Lusaka, Zambia. ${ }^{7}$ Elizabeth Glaser Pediatric AIDS Foundation, Washington, DC, USA. ${ }^{8}$ Department of Obstetrics and Gynecology, Brown University, Providence, RI, USA. ${ }^{9}$ Department of Obstetrics and Gynecology, Columbia University, New York, NY, USA.

Received: 17 December 2018 Accepted: 15 February 2019 Published online: 27 February 2019

\section{References}

1. March of Dimes P, Save the Children, WHO. Born too Soon: the Global Action Report on Preterm Birth. Geneva: WHO;2012.

2. Blencowe $\mathrm{H}$, Cousens $\mathrm{S}$, Oestergaard $\mathrm{MZ}$, et al. National, regional, and worldwide estimates of preterm birth rates in the year 2010 with time trends since 1990 for selected countries: a systematic analysis and implications. Lancet. 2012;379(9832):2162-72.

3. Wedi CO, Kirtley S, Hopewell S, Corrigan R, Kennedy SH, Hemelaar J. Perinatal outcomes associated with maternal HIV infection: a systematic review and meta-analysis. Lancet HIV. 2016;3(1):e33-48.

4. Joint United Nations Programme on HIV/AIDS. The Gap Report. 2014; http:// www.unaids.org/sites/default/files/media_asset/UNAIDS_Gap_report_en.pdf. Accessed 26 Jan 2016.

5. European Collaborative S, Swiss M, Child HIVCS. Combination antiretroviral therapy and duration of pregnancy. AIDS. 2000;14(18):2913-20.

6. Chen JY, Ribaudo HJ, Souda S, et al. Highly active antiretroviral therapy and adverse birth outcomes among HIV-infected women in Botswana. J Infect Dis. 2012;206(11):1695-705.

7. Li N, Sando MM, Spiegelman D, et al. Antiretroviral therapy in relation to birth outcomes among HIV-infected women: a cohort study. J Infect Dis. 2016;213(7):1057-64

8. Townsend C, Schulte J, Thorne C, et al. Antiretroviral therapy and preterm delivery-a pooled analysis of data from the United States and Europe. BJOG. 2010;117(11):1399-410.

9. Zash $\mathrm{R}$, Jacobson DL, Diseko M, et al. Comparative safety of dolutegravirbased or efavirenz-based antiretroviral treatment started during pregnancy in Botswana: an observational study. Lancet Glob Health. 2018:6(7):e804-10.

10. Zash $\mathrm{R}$, Jacobson DL, Diseko $\mathrm{M}$, et al. Comparative safety of antiretroviral treatment regimens in pregnancy. JAMA Pediatr. 2017;171(10):e172222.

11. Society for Maternal-Fetal Medicine Publications Committee waoVB. Progesterone and preterm birth prevention: translating clinical trials data into clinical practice. Am J Obstet Gynecol. 2012;206(5):376-86.

12. Dodd J, Jones L, Flenady V, Cincotta $\mathrm{R}$, Crowther C. Prenatal administration of progesterone for preventing preterm birth in women considered to be at risk of preterm birth. Cochrane Database Syst Rev. 2013;(7):CD004947.

13. Consort 2010. Lancet. 2010;375(9721):1136.

14. Chan AW, Tetzlaff JM, Altman DG, Dickersin K, Moher D. SPIRIT 2013: new guidance for content of clinical trial protocols. Lancet. 2013;381(9861):91-2.

15. Republic of Zambia Ministry of Health. Zambia Consolidated Guidelines or Treatment and Prevention of HIV Infection. Available from: http://www.hivst. org/files1/Final-Zambia-Consolidated-Guidelines_2018-Print.pdf. Accessed 22 Oct 2018.

16. lams JD, Goldenberg RL, Meis PJ, et al. The length of the cervix and the risk of spontaneous premature delivery. National Institute of Child Health and Human Development maternal fetal medicine unit network. N Engl J Med. 1996;334(9):567-72.

17. Villar J, Papageorghiou AT, Knight HE, et al. The preterm birth syndrome: a prototype phenotypic classification. Am J Obstet Gynecol. 2012;206(2):119-23.

18. Vwalika B, Stoner MC, Mwanahamuntu M, et al. Maternal and newborn outcomes at a tertiary care hospital in Lusaka, Zambia, 2008-2012. Int J Gynaecol Obstet. 2017;136(2):180-7.

19. Castillo MC, Fuseini NM, Rittenhouse K, et al. The Zambian preterm birth prevention study (ZAPPS): cohort characteristics at enrollment. Gates Open Res. 2018;2:25
20. Papageorghiou AT, Kemp B, Stones W, et al. Ultrasound-based gestational-age estimation in late pregnancy. Ultrasound Obstet Gynecol. 2016;48(6):719-26.

21. Papageorghiou AT, Kennedy SH, Salomon LJ, et al. International standards for early fetal size and pregnancy dating based on ultrasound measurement of crown-rump length in the first trimester of pregnancy. Ultrasound Obstet Gynecol. 2014;44(6):641-8.

22. Price JT, Winston J, Vwalika B, et al. Quantifying bias between last menstrual period and ultrasonography estimates of gestational age in Lusaka, Zambia. Int J Gynaecol Obstet. 2019;144(1):9-15.

23. Robins JM, Hernan MA, Brumback B. Marginal structural models and causal inference in epidemiology. Epidemiology. 2000;11(5):550-60.

24. Cole SR, Chu H. Effect of acyclovir on herpetic ocular recurrence using a structural nested model. Contemp Clin Trials. 2005;26(3):300-10.

25. Cook NR, Cole SR, Buring JE. Aspirin in the primary prevention of cardiovascular disease in the Women's health study: effect of noncompliance. Eur J Epidemiol. 2012;27(6):431-8.

26. Cain LE, Cole SR. Inverse probability-of-censoring weights for the correction of time-varying noncompliance in the effect of randomized highly active antiretroviral therapy on incident AIDS or death. Stat Med. 2009;28(12):1725-38.

27. Cole SR, Hernan MA. Constructing inverse probability weights for marginal structural models. Am J Epidemiol. 2008;168(6):656-64.

28. Department of Health and Human Services (HHS). Protection of human subjects. Revised rules (45 CFR 46). 2009. Available from: https://www.hhs. gov/ohrp/sites/default/files/ohrp/humansubjects/regbook2013.pdf.pdf. Accessed 16 Oct 2018

29. International Conference of Harmonization (ICH). ICH Tripartite Guideline for Good Clinical Practices E6 (R1), June 10, 1996. Available from: http://www. ich.org/fileadmin/Public_Web_Site/ICH_Products/Guidelines/Efficacy/E6/E6_ R1_Guideline.pdf. Accessed 16 Oct 2018.

30. Meis PJ, Klebanoff M, Thom E, et al. Prevention of recurrent preterm delivery by 17 alpha-hydroxyprogesterone caproate. N Engl J Med. 2003;348(24): 2379-85.

31. Makena. Makena Prescribing Information [package insert]. Available at https://www.accessdata.fda.gov/drugsatfda_docs/label/2013/021945s005lbl. pdf. Accessed 5 May 2015.

32. U.S. Department of Health and Human Services, National Institutes of Health, National Institute of Allergy and Infectious Diseases, Division of AIDS. Division of AIDS (DAIDS) Table for Grading the Severity of Adult and Pediatric Adverse Events, Corrected Version 2.1, July 2017. Available from: https://rsc.niaid.nih.gov/sites/default/files/daidsgradingcorrectedv21.pdf. Accessed 16 Oct 2018.

33. Price JT, Mollan KR, Fuseini NM, et al. Vaginal progesterone to reduce preterm birth among HIV-infected pregnant women in Zambia: a feasibility study protocol. Pilot Feasibility Stud. 2018;4:21.

34. Siou K, Walmsley SL, Murphy KE, et al. Progesterone supplementation for HIV-positive pregnant women on protease inhibitor-based antiretroviral regimens (the ProSPAR study): a study protocol for a pilot randomized controlled trial. Pilot Feasibility Stud. 2016;2:49.

Ready to submit your research? Choose BMC and benefit from:

- fast, convenient online submission

- thorough peer review by experienced researchers in your field

- rapid publication on acceptance

- support for research data, including large and complex data types

- gold Open Access which fosters wider collaboration and increased citations

- maximum visibility for your research: over $100 \mathrm{M}$ website views per year

At $\mathrm{BMC}$, research is always in progress.

Learn more biomedcentral.com/submission 